\title{
The Application of MATLAB in Automatic Fingerprint Recognition System of Police
}

\author{
Hai Ping LI \\ College of Sciences, Hebei University of Science and Technology, Shijiazhuang 050018, China
}

\begin{abstract}
This paper studies on the automatic fingerprint recognition system, the former three fingerprints are extracted from the suspects, the fourth incomplete fingerprint is extracted in scene. Enhance the quality of fingerprint image through improved algorithm and image preprocessing method, at last matching the fingerprint of the three suspects to the fourth to find the criminal. All papers get the simulation results by matlab.
\end{abstract}

\section{Introduction}

Using fingerprint to confirm the identity can be track back to ancient China. At the beginning, fingerprint recognition technology widely applied on the contract, etc, now it began to develop in other areas. The 18th century, Europe's Mayer proposed each person's fingerprints are not the same in the world. In the early 19th century, scientific research found two important fingerprint characteristics: one is the fingerprint ridge lines are different in different finger, another is the pattern of the fingerprint ridge lines will not change all life. Those results were already used in fingerprint recognition of police. In the 20th century, as the computer can process images effectively, it was used to deal with the fingerprint analysis instead of the artificial processing traditionally. By this time, the fingerprint recognition technology reached a comprehensive development stage. At present, fingerprint recognition technology still faces many impediments, especially after the change into a 2 dimension images, some information will be missed, especially the fingerprints extracted is not very complete and clear, for example, lack of some parts, or have scars, twisted, stains, etc, will directly affect the efficiency of fingerprint recognition, which increases the difficulty of feature extraction.

In paper [1], the relative deformations are represented as an average deformation model based on minutiae locations and orientations using 2-D Thin Plate Splines (TPS). Paper[2] shows that the proposed algorithm is more robust and has better binarization performance. In paper [3-5], propounded a new fingerprints matching. Paper [6-7] show that how to enhance the fingerprint image and make it more convenient for the minutiae extraction. In paper [8-10], the thinning algorithm for fingerprint image is studied deeply in these articles. Paper [11-12] introduces a set of algorithms for extracting minutiae from fingerprint image.

\footnotetext{
${ }^{\mathrm{a}}$ Corresponding author: lishuxue@126.com
}

Paper [13-17] discuss preprocessing algorithm in fingerprint recognition.

The rest of this paper is organized as follows: In section 2, preprocessing of the fingerprint image. In section 3, feature extraction of the fingerprint. In section 4 , elimination false of the fingerprints features. In section 5 , matching of the fingerprints.

\section{Preprocessing of the fingerprint image}

The automatic fingerprint recognition system consists of acquisition, preprocessing, feature extraction and matching process fingerprint image, as shown in figure 1.
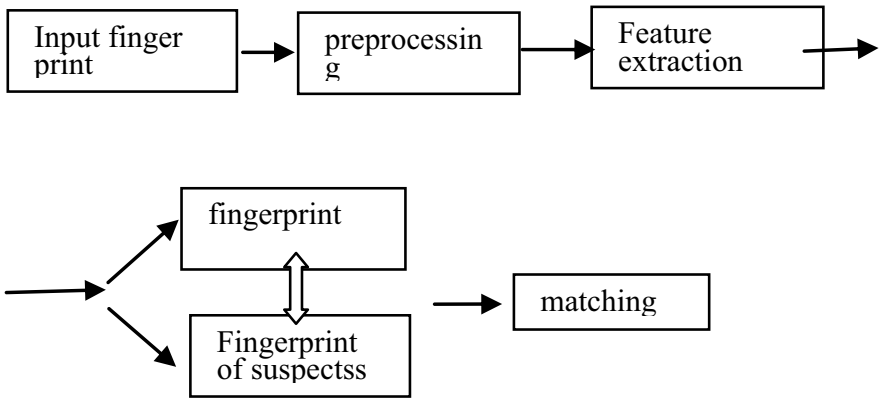

Figure 1. The automatic fingerprint recognition system.

The original fingerprint images are shown in figure2:
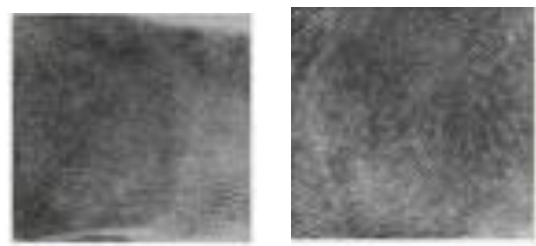


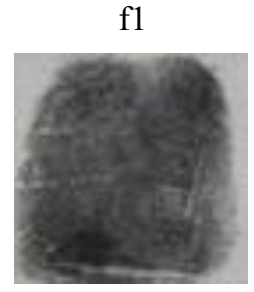

f3

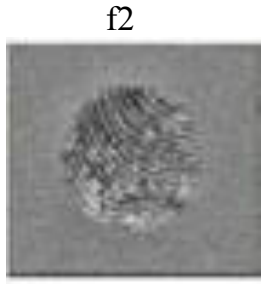

f4
Figure 2. Original fingerprint image.

It is difficult in the extraction of fingerprint in fingerprint recognition system of police, so high demands in the preprocessing of the fingerprint system than in the company's fingerprint attendance system. Image preprocessing is the key in the fingerprint identification, it includes: grayscale transformation, binarization, filtration (noise reduction) and refinement.

(1) Grayscale transformation: using matlab's function rgb2gray, the results are shown in figure 3 .

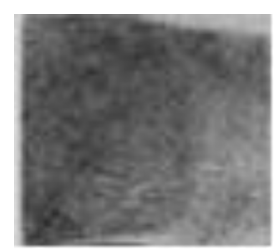

f1

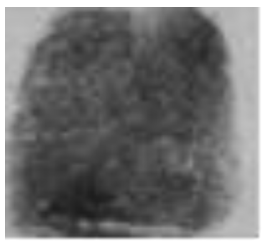

f3

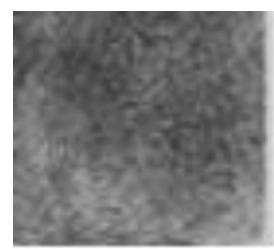

f2

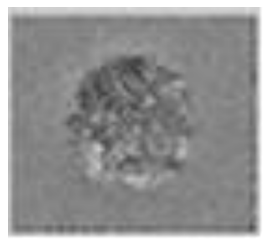

f4
Figure 3. Grayscale of fingerprint image.

(2) The median filtering: median filtering is a pixel grayscale by the adjacent field, output the median pixels (here can also use the median filter). The greater median filtering window, the stronger filtering effected. But with the increase of the window, some of its details will be losed. The $(4 * 4)$ median filtering is a nonlinear filtering, suitable the nose of granular type and can protect the edge of the image, the results are shown in figure 4.

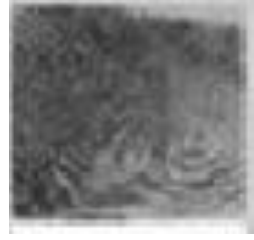

f1

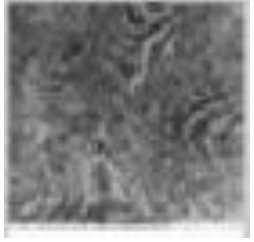

f2

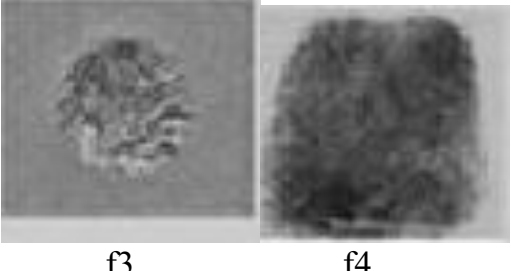

f3

Figure 4. Denoising of fingerprint image.

(3) Binarization: fingerprint image binarization is to compare each pixel, if greater than the threshold value, let the value is 1 ; if less than the threshold, let the value is 0 . After binarization, not only can reduce the amount of data greatly, and the make less interference of the process behind, to simplify the processing greatly, the results are shown in figure 5 .

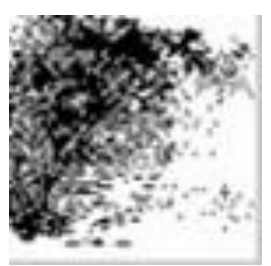

f1

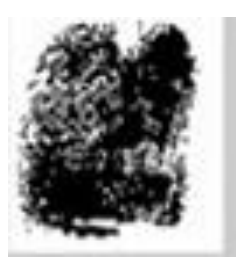

f3

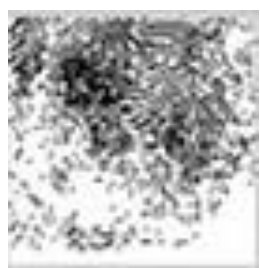

f2

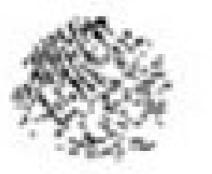

f4

Figure 5. Binarization of fingerprint image.

(4) Thinning: fingerprint thinning is reduced the width of crest to a single pixel, get the skeleton of ridge line image process. This process further reduces the data quantity of fingerprint image, clear the form of the crest line, prepared for the feature extraction of the fingerprint. Using matlab function "bwmorph" carries on the thinning, the results are shown in figure 6 :

$\mathrm{s} 11=$ bwmorph(f13, 'skel',Inf); This statement is to extract the bones of the fingerprint image.

$\mathrm{s} 1=$ bwmorph(s11, 'spur',8); This statement is to delete the burr extracted from fingerprint image skeleton.

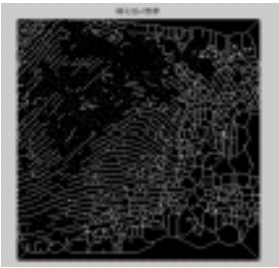

f1

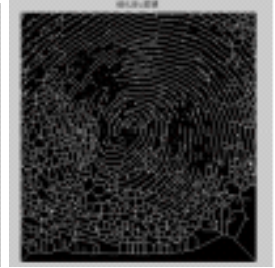

$\mathrm{f} 2$ 


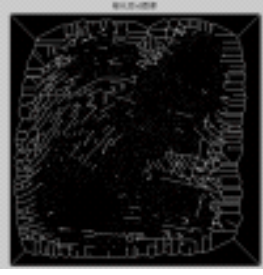

f3

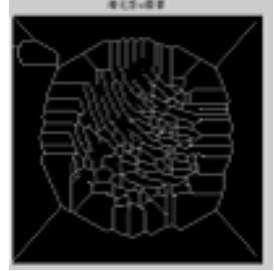

f4
Figure 6. Thinning of fingerprint image.

\section{Feature extraction of the fingerprint}

Extract the feature points from the elaborated binary image to get the useable image, and then extract the feature point by using the improved image enhancement algorithm, at last matching the thinned fingerprint in the template.

Feature points have six types before the feature point extraction: endpoints, branch points, bifurcation, isolated points, ring and short grain. True and false of the feature points will affect the effect of matching directly, we often do post-processing after feature extraction to eliminate the false feature points, this is what we called the pseudo features. Since there are so many fingerprint characteristics, we only test the feature of bifurcation point and endpoint respectively in this simulation. Considering some information may be missed after the thinning, we use eight neibourhood algorithm which is the template matching method, but we has made great improvement in the process. The eight neibourhood method to extract feature points is shown in figure 7 :

\begin{tabular}{|l|l|l|}
\hline N4 & N3 & N2 \\
\hline N5 & $(\mathrm{x}, \mathrm{y})$ & $\mathrm{N} 1$ \\
\hline N6 & N7 & N8 \\
\hline
\end{tabular}

Figure 7. Eight neibour table.

After fingerprint image change from the gray image into binary image, take point $(\mathrm{x}, \mathrm{y})$ for example, judge it is effective pixels or not based on its around eight points in the neighborhood. If the "0", "1" change six times, then it is the bifurcation point; if change twice, it is endpoint. The main algorithm is described as follows:

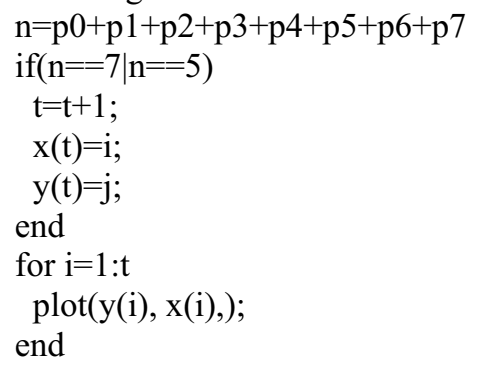

\section{Elimination false of the fingerprints feature}

Elimination false is mainly to remove the fingerprint feature points which do not conform to the characteristics. The false features most in the edge of the fingerprint image, especially as we can see from $\mathrm{f} 4$ which is after refining and extraction of characteristic value that the edge of the fingerprint image is all pseudo features. According to these features, we put forward a new method of pseudo: first cut the edge of the image directly; then remove the feature points closed based on distance threshold value method. The main algorithm is described as follows:

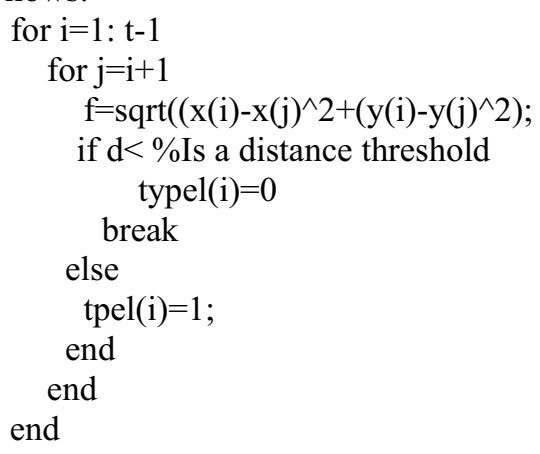

\section{Matching of the fingerprints}

Observe from f4: the most effective information concentrated in the lower right part of the fingerprint image after elimination false features of $\mathrm{f} 4$, thus interception the v4 fingerprint feature image using matlab, clockwise rotation 30 degrees respectively and then matching with the three fingerprints, the purpose is to improve the matching rate. The interception of information is shown as follows in Figure 8.

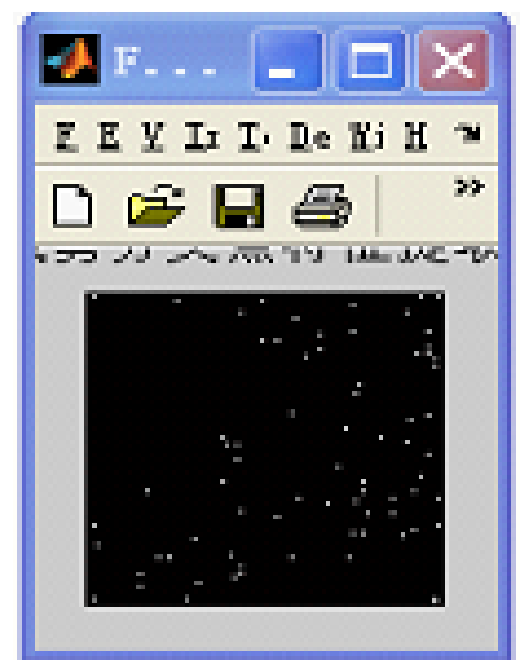

Figure 8. F4 intercept information graph.

By using the similarity of the two matrix, comparing matrix elements with the same position:

ht211=imcrop(ht2, [I, j, 9, 99]);

$2=$ length(find(ht $211-\mathrm{ht} 4==0) ; \%$ two matrix similarity. 
Stop when two matrix have similarity $99 \%$ and timing the used time by matching, when the program met "tic", automatically start timing by Matlab, when run to "toc" automatic computing the time between the last "tic", the matching result is shown in figure 9 .

Command Window
Elapsed time is 15.148022 seconds.
Elapsed time is 14.989565 seconds.
natch with f3

Figure 9. Matching.

\section{Conclusion}

This paper puts forward some different algorithms and Matlab simulations, simplifies the complexity of preprocessing, improves the accuracy of the feature extraction, strengthens the accuracy of matching.

The results show that the algorithm need long time to the mass of fingerprint recognition system, but have a much higher accuracy for the police. Though enhance the image by the algorithm, fingerprint minutiae can be extract accurately. The false feature points which caused by various reasons removed by different algorithms respectively. These algorithms not only do effect the precision of matching, but also improve the speed of fingerprint image matching recognition and performance.

\section{References}

1. Chen, Y.,Dass, S.,Ross, A.,\&Jain, A. Fingerprint deformation models using minutiae locations and orientations. Appeared in Proc. of IEEE Workshop on Applications of Computer Vision (WACV), (Breckenridge, Colorado), 150-156.(2005)

2. Ji,C., Feng, W., Li, M., \&Yang, J. Dynamic Threshold with Hole Padding Algorithm for Fingerprint Image BinariZation.Computer Simulation, 28(7), 258-261. (2011)

3. Mayur D Jain, Nalin Pradeep S, Prakash C and Balasubramanian Raman, Binary tree based linear time fingerprint matching.309312.(2006)

4. Liu,Y.,Cao, J., Xu,Z.,Tian,Y.,Fu, T., \&Wang,F., Improvement of image matching algorithm based on gray correlation.Journal of Applied Optics. 28(5), 536-540.(2007)

5. Zhao,J., \&Wang, D.The implementation of a fingerprint matching algorithm. Computer Engineering and Applications,13, 66-69. (2005)

6. Wang,J.,Chen,S., \&Sun,S., Realization of the fingerprint image enhancement based on matlab. Electronic Science and Technology. 6,37-39.(2009).

7. Wang,Y., \&Su,C., Study of fingerprint image enhancement algorithm. science technology and Engineering,10(1),9498.(2010)

8. Wang,Y.,Ning,X., \&Yin,Y., Study on the fingerprint image thinning algorithm. Journal of Nanjing University (Natural Sciences), 39(4), 468-475.(2003)

9. Xiao,X., Wang,K., \&Li,Z., mproved thinning method to fingerprint image. Computer Applications, 28(2), 466-468.(2008)

10. Yang,W., Guo, K., \&Wei,Y., An efficient index thinning algorithm of fingerprint image based on eight neighborhood point. Journal of Sichuan University of Science\& Engineering (Natural Science Edition), 21(2), 61-63.(2008)

11. Guo,J.,Wu,Q., \&Shang,Q., Minutiae extraction of fingerprint image based on matlab. Computer Simulation,24(1),182185.(2007)
12. Liao,K.,Zhang,X., Zhang,M.,\&Pan X, Extraction of minutiae and significant feature from fingerprint image. Computer Applications, 28(9), 2312-2314.(2008).

13. Shang,Q., Wu, Q., \&Guo,J. Preprocessing algorithm of fingerprint image based on matlab. Computer Simulation, 24(3), 215-218.(2007)

14. Tai,Y. Fingerprint processing algorithm based on matlab. Journal of Southwest University for nationalities (Natural Science Edition), 34(4), 836-838.(2008).

15. Wu,J.,Xu,C.,Yang,K.,\&Zhang,G., Study of essential technology for fingerprint image Pre-processing.Computer Engineering and Applications, 44(3), 223-239.(2008)

16. Xue,J.,Wang,S., \&Liu,Z., Adaptive pre-processing for fingerprint image. Computer Engineering and Design, 29(1), 157-159.(2008)

17. Ye,Q. Study of a fingerprint pre-processing and matching algorithm. Journal of North China University of Technology Beijing China, 24(3) , 6-10.(2012).

18. Wang Guwen, Cao Yu MATLAB6. 5 graphics image processing [M]. Beijing: national defence industry press, ( 2004

19. RuanQiuqi. Digital image processing [M]. Beijing: electronic industry press, (2007) 